Mots. Les langages du politique

\title{
La construction de l'image des hommes politiques par le folklore narratif. Anecdotes, rumeurs, légendes, histoires drôles
}

Jean-Bruno Renard

\section{(2) OpenEdition \\ Journals}

Édition électronique

URL : https://journals.openedition.org/mots/19418

DOI : $10.4000 /$ mots. 19418

ISSN : 1960-6001

Éditeur

ENS Éditions

\section{Édition imprimée}

Date de publication : 1 mai 2010

Pagination : 11-22

ISBN : 978-2-84788-209-4

ISSN : 0243-6450

\section{Référence électronique}

Jean-Bruno Renard, « La construction de l'image des hommes politiques par le folklore narratif. Anecdotes, rumeurs, légendes, histoires drôles », Mots. Les langages du politique [En ligne], 92 | 2010 , mis en ligne le 04 mai 2012, consulté le 23 avril 2022. URL : http://journals.openedition.org/mots/ 19418 ; DOI : https://doi.org/10.4000/mots.19418 


\section{La construction de l'image des hommes politiques par le folklore narratif. Anecdotes, rumeurs, légendes, histoires drôles}

Ce texte est consacré au folklore narratifi ${ }^{1}$ qui prend pour objet les hommes politiques. Ni étude de cas, ni réflexion théorique, il a l'ambition de montrer le profit heuristique que l'on peut tirer - si l'on s'intéresse aux langages du politique - de la collecte et de l'analyse de ces «petites histoires».

"À l'aide de trois anecdotes, on peut faire le portrait d'un homme.» (Nietzsche, 1951, p. 27) Cette intuition nietzschéenne trouve une illustration dans l'étude de deux historiens d'art autrichiens, Ernst Kris et Otto Kurz (1987), sur l'image de l'artiste. Collectant les anecdotes que l'on rencontre dans les biographies des peintres et des sculpteurs, les auteurs ont mis en évidence non seulement l'image qui est donnée de chaque artiste, mais, plus encore, la construction de l'image (ou des images) de l'artiste type, au travers d'anecdotes stéréotypées qui révèlent des constantes thématiques. Par exemple le thème, très fréquent, de l'artiste qui imite la nature à s'y méprendre :

- Giotto aurait peint une mouche sur une œuvre de son maitre, lequel tenta de la chasser avant de s'apercevoir de son erreur;

- selon la biographie de Raphaël, un cardinal tendit un jour par méprise une plume et un encrier au portrait si ressemblant de Léon X afin d'obtenir la signature du pape.

Les auteurs relèvent bien d'autres thèmes tels que le génie précoce, l'orgueil de l'artiste, son obsession du travail, ses vengeances contre des commanditaires mauvais payeurs, sa rivalité avec d'autres artistes, etc.

D'ailleurs, l'existence de leitmotive biographiques était déjà bien connue des folkloristes spécialisés dans les légendes hagiographiques, mais c'était la première fois que l'on appliquait le même regard à des personnages laïcs.

1. Nous distinguerons le «folklore narratif» de la «littérature folklorique», qui recouvre les mythes, légendes, contes, fables, fabliaux. Le premier domaine est peu structuré et correspond à de «petites histoires» disséminées, alors que le second est plus structuré et désigne des ensembles de récits - mythologies ou répertoires de contes - souvent formalisés dans des écrits. Naturellement, des motifs de la littérature folklorique (voir Thompson, 1989) peuvent se retrouver dans le folklore narratif. 
Tout laisse à penser que des études semblables pourraient être menées sur les anecdotes biographiques de savants, de héros de la guerre, de sportifs, de personnalités du spectacle et, bien évidemment, d'hommes politiques.

Nous examinerons comment appliquer cette approche aux personnalités politiques à travers quelques principes méthodologiques.

\section{La variété du folklore narratif}

Nous proposons tout d'abord de ne pas se limiter auxanecdotes, mais d'élargir les données à toutes les formes du folklore narratif :

- les anecdotes sont des récits de petits évènements prétendument historiques qui mettent en évidence, à travers un trait psychologique (générosité, courage, ruse, bêtise, orgueil, etc.), le caractère d'un individu célèbre ou d'une classe d'individus (les aristocrates, les bourgeois, etc.), ou encore les mœurs d'une époque (libertinage, cruauté, etc.) ;

- les rumeurs sont des énoncés qui prétendent révéler des informations nouvelles, importantes et encore peu connues, concernant un sujet, que celuici soit un individu, un groupe social ou ethnique ou un objet matériel (Kapferer, 1990);

- les légendes urbaines sont des rumeurs narratives qui ressemblent à de petits faits divers (Renard, 2006a). Leurs protagonistes sont le plus souvent anonymes. Si elles concernent des célébrités, elles sont indiscernables des anecdotes;

- les propos attribués («mots historiques») rapportent non des actions de célébrités mais des phrases, d'allure proverbiale, qui révèlent de manière imagée les idées ou les sentiments de leurs auteurs;

- les histoires drôles sont des histoires inventées, concernant des célébrités ou des anonymes, et destinées à faire rire, en particulier par leur chute inattendue;

- les blagues sont un type d'histoires drôles généralement construites sur une question («Pourquoi Untel... ?») dont la réponse recèle un trait d'esprit.

Les diverses formes du folklore narratif partagent des caractéristiques communes : une origine indéterminée; une forme brève; une circulation de bouche à oreille (ou aujourd'hui par Internet); l'existence de variantes qui marquent l'adaptation de ces "petites histoires » aux époques et aux milieux successifs de leur diffusion; un contenu surprenant, insolite ou comique; last but not least, toutes ces formes narratives contribuent à la construction d’images stéréotypées, orientées vers une dévalorisation ou une valorisation de leur objet.

On peut voir une preuve de l'unicité du champ du folklore narratif dans la fréquence des passages d'une forme à l'autre : ainsi une anecdote biogra- 
phique exacte ou un propos réellement tenu par une célébrité peuvent, ultérieurement, être faussement attribués à une autre personne, ou bien un récit légendaire au contenu humoristique peut aisément devenir une histoire drôle². Par exemple, le motif du «président qui se noie» est attesté en tant que légende urbaine :

On raconte qu'un jour le président Richard Nixon, en train de se baigner dans l'océan face à sa propriété de San Clemente en Californie, fut en danger de se noyer. Deux jeunes gens qui faisaient du surf se précipitèrent et le sauvèrent. Pour les remercier, il leur demanda ce qu'ils souhaitaient. Le premier émit le souhait que la plage privée réservée au président fût ouverte au public afin que chacun pût en profiter. Nixon promit que cela serait fait. Le second surfeur demanda s'il pouvait avoir une tombe pour lui-même au cimetière national d'Arlington. Le président dit que cela pouvait être fait, mais il demanda au jeune homme pourquoi il avait formulé un souhait aussi insolite. Le surfeur répondit alors : «Parce que, lorsque mon père apprendra que j'ai sauvé la vie de Richard Nixon, il me tuera!» (D'après Brunvand, 1993, p. 199)

Cette légende était déjà racontée dans les années 1959-1960 en Finlande à propos du président Urho Kekkonen. Le même motif, appliqué cette fois-ci à George W. Bush, circule comme histoire drôle sur Internet depuis 2004 sous la forme d'un pseudo-test:

Ce test n'a qu'une seule question, mais c'est une question très importante. En donnant une réponse honnête et spontanée, vous découvrirez votre attitude morale. Le test propose une situation fictive dans laquelle vous aurez à prendre une décision. Vous êtes en Floride, au milieu d'un chaos causé par un ouragan avec de graves inondations. Vous êtes photographe et travaillez pour un journal important. Vous tentez de faire des photos. Il y a des maisons et des personnes qui tourbillonnent autour de vous puis disparaissent sous l'eau.

Soudain, vous voyez dans l'eau un homme qui se débat et qui risque d'être englouti. Vous réalisez soudain que c'est George W. Bush en personne!

Vous avez deux options : vous pouvez sauver la vie de Bush ou vous pouvez prendre une photo dramatique qui vous fera gagner le Prix Pulitzer, la photo de la mort de l'un des hommes les plus puissants.

Voilà la question, et merci de donner une réponse honnête : prendrez-vous une pellicule en couleur, ou bien choisirez-vous la simplicité classique du noir et blanc?

Étudier les rumeurs sur un homme politique en négligeant les histoires drôles qui se racontent sur lui conduit à se priver de matériaux qui viennent compléter et souvent éclairer les données déjà recueillies. Le fait de considérer

2. Il n'est pas rare que des légendes urbaines comiques passent du registre des histoires racontées comme vraies au registre des histoires drôles, qui sont d'abord destinées à faire rire et dont la véracité importe peu. Par exemple, l'histoire de l'éléphant qui s'assied sur une petite voiture parce qu'il la confond avec son tabouret de cirque est racontée comme authentique en Angleterre et en Allemagne, mais comme une histoire drôle en France en raison du célèbre sketch qu'en a tiré Fernand Raynaud, «La 2 CV de ma sœur» (Renard, 2006a, p. 69). 
comme équivalentes les différentes formes du folklore narratif a deux conséquences méthodologiques importantes que l'on va examiner dans les sections suivantes : d'une part, on contourne ainsi l'épineuse question de la véracité des données narratives et, d'autre part, on met en évidence le phénomène de convergence des différentes formes du folklore narratif dans la construction d'une même image.

\section{La question de la véracité}

Le folklore narratif navigue constamment entre le vrai et le faux. Même les anecdotes biographiques historiquement attestées n'offrent qu'une vérité partielle et partiale: l'anecdote a été sélectionnée parmi de multiples petits évènements 3 parce qu'elle est considérée comme emblématique d'un trait de caractère que l'on veut souligner, au détriment de petits faits biographiques tout aussi exacts mais qui prouveraient un autre trait de caractère, voire même un trait de caractère opposé. D’ailleurs, le plus souvent, les anecdotes sont fausses ou partiellement fausses. Pour le Grand dictionnaire universel du xIXe siècle, "l'histoire sérieuse est tenue de se défier des anecdotes, parce que ce ne sont bien souvent que des récits faits à plaisir » (Larousse, 1866, t. I, p. 345, col. 1).

De même, le terme rumeur possède deux sens :

- la rumeur comme information non vérifiée. À ce stade, on ne préjuge pas de la véracité du «bruit qui court» et, dans cette acception, une rumeur peut se révéler exacte : par exemple la maladie ou la fille cachée d'un président de la République ;

- La rumeur comme information fausse. Si, après vérification, une information se révèle inexacte, nous sommes alors en présence d'une rumeur au second sens du terme, c'est-à-dire une «fausse nouvelle», à laquelle les gens ont cru ou croient encore.

Rien ne permet a priori de distinguer une rumeur vraie d'une rumeur fausse.

L'étude des légendes urbaines, qui sont généralement fausses, ne serait-ce qu'en raison des multiples variantes, datations et localisations du récit, ne saurait nous conduire à exclure que l'évènement raconté ait pu un jour avoir eu lieu : par exemple, la découverte d'un doigt coupé dans la nourriture ou le séchage au four à micro-ondes d'un petit animal domestique.

L'établissement de la véracité ou de la fausseté d'une rumeur présente un intérêt historique ou bien répond à la demande légitime de démenti de la part de la victime d’une fausse rumeur, mais ce travail de vérification est secondaire

3. On pourrait aisément montrer que les faits divers dans la presse sont le résultat d'un même processus de sélection et d'écriture de la réalité. 
si l'on cherche à comprendre pourquoi les gens diffusent ces histoires, c'està-dire si l'on s'intéresse à la signification des anecdotes et aux motivations de ceux qui les diffusent. Si une même histoire peut être racontée comme une anecdote véridique ou comme une histoire drôle, c'est bien la preuve que « la signification importe plus que l'exactitude factuelle» (Gritti, 1978, p. 65). Ces «petites histoires» circulent non pas tant parce qu'elles sont crues vraies que parce qu'elles sont «exemplaires» (Campion-Vincent, 1976), c'est-à-dire qu'elles expriment en peu de mots, à travers une situation concrète - et peu importe si celle-ci est fausse - une idée à laquelle on adhère et que l'on veut partager.

\section{L'effet de convergence}

La construction de l'image d'une célébrité politique s'appuie sur la convergence thématique de formes diverses du folklore narratif qui aboutissent à brosser un portrait particulier du personnage. Vraies ou fausses, ces formes narratives mettent l'accent sur des traits de caractère ou de comportement. Gordon Allport et Leo Postman (1965), pionniers de l'étude scientifique des rumeurs, ont précisément identifié l'«accentuation » (sharpening en anglais) comme l'un des mécanismes fondamentaux de distorsion de l'information. Par exemple, des individus dont la taille est plus petite que la moyenne deviendront des nains, et ceux dont la taille est plus grande deviendront des géants. C'est le même procédé qui préside à la caricature : que l'on pense aux dessins qui accentuent des traits physiques comme la grande taille du général de Gaulle, la petite taille de Michel Rocard, les sourcils broussailleux de Leonid Brejnev... Il en est de même pour les traits psychologiques ou moraux : un économe devient un avare, un nerveux un agité, etc. L'accentuation peut aussi porter sur le nombre : une assemblée d'une vingtaine de personnes sera transformée en foule par la rumeur; une somme d'argent deviendra une fortune; le nombre de morts et l'ampleur des dégâts matériels causés par une catastrophe seront encore augmentés... On a bien là un mécanisme fondamental des rumeurs. Et si la réalité ne fournit pas l'évènement - même mineur - qui pourra servir de germe à une exagération, l'imaginaire collectif l'inventera!

Jules Gritti a été l'un des premiers à souligner que les rumeurs politiques, dans lesquelles il inclut d'ailleurs les «mots historiques», opèrent « une figuration d'un personnage» (Gritti, 1978, p.65). Jean-Noël Kapferer, reprenant cette idée, montre "comment on crée une image » à travers les rumeurs politiques (Kapferer, 1990, p. 255-259). Une «cible», c'est-à-dire l'homme politique visé, est l'objet de «rumeurs types» qui contribuent à la construction d'une « image ». Par exemple, Valéry Giscard d'Estaing a fait l'objet de rumeurs d'escapades nocturnes et de liaison avec une actrice en vue qui donnent de lui une image d'époux volage. D'autres rumeurs racontaient qu'il aurait été 
giflé (rumeur du laitier4 et rumeur de la gifle de Michel Piccoli5), forme allégorique de vengeance populaire contre un homme jugé hautain, jugement que renforçaient encore les connotations symboliques de sa grande taille et de son patronyme à particule. Enfin, l'affaire des diamants de Bokassa 6 et la rumeur selon laquelle Giscard possédait des forêts domaniales en Grèce le montraient comme un homme avide de richesses. Il résulte de tout cela le portrait d'un «Roi léger» (Kapferer, 1990, p. 257), hautain et égocentrique, semblable au Régent ou à Louis XV. Tout autre est le portrait de François Mitterrand, brossé par l'affaire de l'attentat de l'Observatoire?, la rumeur de cancer, la découverte d'une fille cachée, l'affaire des «écoutes de l’Élysée» (Mitterrand aurait écouté les conversations téléphoniques d'un certain nombre de personnalités), la signification occulte des monuments qu'il a fait construire à Paris (Pyramide du Louvre, Arche de la Défense) : l'image qui s'en dégage est celle d'un monarque usé, rusé et secret.

L'effet de convergence a été théorisé par Michel-Louis Rouquette (1989), qui considère que les rumeurs sont comme des «solutions » à des «problèmes » et qu'il existe infiniment plus de solutions que de problèmes. Derrière la prolifération des rumeurs, on peut identifier un petit nombre de motivations sociales qui les ont suscitées. "Il existe donc moins de "problèmes" générateurs que de rumeurs correspondantes; de ce point de vue, celles-ci forment des classes d'équivalence qu'il serait sans doute possible de reconstituer inductivement » (Rouquette, 1989, p.121). Cette position méthodologique présente l'avantage de ne pas se noyer dans le corpus ouvert et infini des rumeurs pour se concentrer sur les problèmes, ici les images d'hommes politiques.

On voit que les rumeurs sont indépendantes de tout critère de vérité : certaines sont fausses (la gifle de Michel Piccoli), d'autres se sont révélées vraies (le cancer de Mitterrand et sa fille illégitime); pour d'autres encore, la vérité historique n'a pas été clairement établie (l'attentat de l'Observatoire). C'est pourquoi nous suggérons d'élargir le champ des rumeurs et d'y inclure les «mots historiques» et les « histoires drôles».

Citons, en guise d'illustration, la réputation de bêtise de George W. Bush, déclinée selon divers registres ${ }^{8}$ :

4. Valéry Giscard d'Estaing, alors président de la République, « rentrant à l'aube après une escapade nocturne, au volant de la voiture de sport de son ami Vadim [...], aurait renversé le camion d'un laitier qui passait par là. Une altercation aurait suivi et le laitier l'aurait giflé » (Kapferer, 1990, p. 255-256).

5. Lors d'une réception officielle, Michel Piccoli aurait giflé Giscard parce qu'il n'organisait pas la libération de $\mathrm{M}^{\mathrm{me}}$ Claustres, prisonnière au Tchad.

6. En 1979, on prétendit que le président centrafricain Bokassa avait donné en 1973 au président Giscard d'Estaing une plaquette de diamants.

7. La nuit du 15 au 16 octobre 1959, François Mitterrand échappa à un attentat près des Jardins de l'Observatoire à Paris. On prétendit qu'il avait lui-même organisé un faux attentat afin de retrouver une popularité qu'éclipsait celle du général de Gaulle.

8. Sauf exception, on ne référencera pas les anecdotes dont on trouve de multiples citations et 


\section{- Propos rapportés:}

- mots écorchés : «ll faut garder de bonnes relations avec les Gréciens. » (La «bravitude » de Ségolène Royal, même si ce mot malheureux a été largement exploité, n'a pu susciter une image de bêtise en raison de la réputation de femme intelligente et instruite dont bénéficie cette personnalité);

- lapsus : "Le roi Abdallah de Jordanie, roi du Maroc »;

- lapalissades: «l'ai une politique étrangère axée sur l'étranger» 9 ;

- phrase d' "arroseur arrosé» : «Je suis convaincu que si vous savez en quoi vous croyez, il est alors beaucoup plus facile de répondre aux questions. Je ne peux pas répondre à votre question. $»^{10}$

- Comportement : une vidéo a largement circulé, montrant Bush dans une classe d'enfants et tenant à l'envers un livre entre ses mains.

- Rumeur : une rumeur prétendit que lors de ses débats télévisés contre John Kerry, en 2004, Bush était équipé d'un récepteur radio camouflé sous son costume (un pli suspect en témoignait!) afin que ses conseillers puissent lui souffler des réponses intelligentes.

- Histoire drôle :

Un avion est sur le point de se crasher. Il y a cinq personnes à bord et seulement quatre parachutes. Le premier passager dit: «Je suis Kobe Bryant, le meilleur joueur de basket au monde. Les LA Lakers ont besoin de moi. Je ne peux pas mourir. " II prend un parachute et saute.

Le second passager, Hillary Clinton, dit : "Je suis la femme de l'ex-président des USA. Je suis aussi la femme la plus ambitieuse sur terre et une présidente potentielle des USA... » Elle prend le second parachute et saute.

Le troisième passager, George W. Bush dit : «Je suis le président des USA. Je suis le leader du monde libre et de la plus grande nation du monde. Et plus encore, je suis le président le plus intelligent de l'Histoire américaine. » Il endosse le troisième parachute et saute.

Le quatrième passager, le Pape, dit au cinquième passager, un petit écolier de 10 ans: "Je suis vieux et malade et il ne me reste pas beaucoup de temps sur terre, de toute façon. En tant que catholique, je te donne le dernier parachute... » Le gamin répond: «Non, c'est bon, il reste un parachute pour vous. Le président le plus intelligent de l'Histoire américaine a pris mon cartable... »

Cette grille, qui liste les types d’énoncés (propos, comportements réels, rumeurs et légendes, histoires drôles) contribuant à la construction d'une

variantes, en particulier sur les sites Internet d'humour, de citations, d'anecdotes. Rappelons que notre objectif n'est pas d'établir l'authenticité d'une anecdote, mais de montrer son rôle dans la construction de l'image des hommes politiques.

9. Cela rappelle la célèbre exclamation attribuée à Mac-Mahon - dont les déclarations ou «macmahonneries» annoncent les «busheries» - lorsqu'il vit, en juin 1875, les terribles inondations de la Garonne: "Que d'eau! Que d'eau! Et encore, on ne voit que le dessus.»

10. De même, Mac-Mahon aurait déclaré : «La fièvre typhoïde est une maladie terrible. Ou on en meurt, ou on en reste idiot. Et je sais de quoi je parle, je l'ai eue.» 
image stéréotypée, pourrait aisément être appliquée à d’autres traits psychologiques et à d'autres personnalités politiques.

\section{Motifs et thèmes du folklore narratif politique}

La distinction entre motif et thème a été avancée par le folkloriste Arthur Christensen (1925). Le motif est une configuration narrative brève et stéréotypée - les littéraires parlent de topos - qui s’incarne dans de multiples variantes : par exemple, le motif de l'insecte peint sur une toile déjà terminée (voir plus haut), le motif de la bestiole dangereuse cachée dans un produit exotique dans les supermarchés (motif récurrent des légendes urbaines), le motif des impôts non payés par un homme politique ou celui de son appartenance à une société secrète. Le thème est «l'idée fondamentale exprimée par un motif ou un assemblage de motifs» (Christensen, 1925, p. 8). C'est la signification morale ou psychologique du motif, "l'image à construire» selon Kapferer. En référence aux motifs énoncés ci-dessus, on associera le thème de l'art qui imite la nature, celui de la peur de l'étranger et des risques de la société de consommation, et les thèmes politiques de l'abus de pouvoir et de «la main cachée ». Le motif est concret, descriptif, alors que le thème est abstrait, interprétatif.

Jean-Noël Kapferer (1990, p. 251-255) a identifié sept thèmes récurrents des rumeurs politiques : le pouvoir occulte caché derrière le pouvoir officiel; l'accord secret entre adversaires politiques; l'argent ; la santé ; la sexualité ; le double langage; la connivence avec l'étranger. Il y en a d'autres, comme l'arrogance, la trahison, la bêtise...

Depuis les anecdotes racontées par Suétone sur les empereurs romains jusqu'aux rumeurs d'aujourd'hui concernant les chefs d'État, il existe toute une tradition du folklore narratif sur les mœurs sexuelles de nos dirigeants, y compris dans le répertoire d'histoires drôles, comme en témoigne par exemple le grand nombre de blagues - suite à l'affaire Lewinsky - concernant Bill Clinton, dont l'infidélité conjugale est poussée jusqu'à l'absurde : «On a fait un sondage aux États-Unis. On a demandé aux femmes si elles voulaient faire l'amour avec Bill Clinton. 99\% des femmes ont répondu : "Non, pas encore une fois!" "

L'image de Bill Clinton, toujours en raison de la même affaire, est aussi associée au mensonge. D’où cette histoire drôle :

C'est un gars qui arrive au paradis et qui voit saint Pierre. Celui-ci lui fait visiter un peu tout et ils arrivent dans une pièce où y a plein de montres. Le gars étonné demande ce que c'est. Saint Pierre lui répond : «Ce sont les montres des gens qui vivent encore. Plus on ment, plus les aiguilles tournent vite.» Le gars s'avance et perçoit un fort courant d'air : «C'est quoi ça?» Réponse immédiate de saint Pierre : «Ah, ça, c'est la montre de Bill Clinton, on s'en sert comme ventilateur... » 
Une version plus récente de l'histoire remplace les montres par des horloges qui correspondent à des célébrités diverses et qui avancent d'une heure "chaque fois que son propriétaire dit une connerie» : l'horloge qui sert de ventilateur est celle de G. W. Bush.

Une autre tradition narrative exploite le thème des caprices d'autocrates, depuis Caligula nommant son cheval sénateur jusqu'à Ceausescu ordonnant cinq fois de suite de détruire et de reconstruire l'escalier monumental en marbre du Palais présidentiel de Bucarest jusqu'à ce que la hauteur des marches corresponde à son pas.

On trouve aussi des anecdotes ou des histoires drôles qui ironisent sur le prétendu sentiment de toute-puissance des hommes politiques ${ }^{11}$ :

Un jour que le roi Hassan II du Maroc inaugurait un barrage, sous trois grandes enseignes lumineuses portant chacune un mot de la devise du royaume, «Dieu, la Patrie, le Roi », un incident technique nécessita de disposer de plus d'électricité et l'on demanda au roi quelle enseigne pouvait être éteinte. Hassan II répondit : «Dieu. Cela ne le dérangera pas!»

Jacques Chirac, Dominique de Villepin et Nicolas Sarkozy arrivent au paradis. Ils sont reçus par Dieu lui-même, qui leur demande à tour de rôle : «En quoi crois-tu? » Chirac fait une profession de foi écologiste et Dieu le fait asseoir à sa gauche. Villepin dit qu'il croit en la bonté de l'homme et Dieu le fait asseoir à sa droite. Puis Dieu s'adresse à Sarkozy et lui demande : «Et toi, Nicolas, en quoi crois-tu?» Et Sarkozy répond : "Je crois que vous êtes assis à ma place! »

De manière plus euphémique, des «mots historiques» confèrent une image élitiste à des hommes politiques de caractère. Ainsi Churchill - dont le répertoire de mots d'esprit est riche - aurait déclaré : «Un chameau est un cheval proposé par une commission. » On connait aussi la célèbre réplique du général de Gaulle qui, voyant écrit sur un mur «Mort aux cons!», se serait écrié: «Vaste programme!»

S'il n'est pas surprenant qu'un même thème puisse être associé à des hommes politiques différents, on observe aussi qu'un même motif, parfois exactement la même variante, peut être attribué à des personnalités différentes. C'est particulièrement le cas des «mots historiques». Citons l'histoire de l'homme politique qui, visitant le Collège de France à Paris, demande naïvement: «Où sont les dortoirs? » Ces propos ont été attribués successivement à un ministre de la Restauration, puis de la République, et enfin à Mac-Mahon (Gaidoz, 1898-1899, col. 77 et col. 118). Cette anecdote avait pour but de ridiculiser un homme politique que l'on jugeait peu instruit ou peu intelligent.

Le sociologue Gary Alan Fine (1985) a appelé « effet Goliath» la tendance d'une rumeur concernant un type d'objet à se fixer sur l'objet le plus connu du moment : par exemple, les rumeurs sur les hamburgers ou sur les blue-jeans

11. On se souvient que les humoristes français surnommaient «Dieu» le président Mitterrand! 
prendront tout naturellement pour cible les restaurants McDonald's et les jeans Levi's. Ainsi la célèbre réplique de la reine Marie-Antoinette - «lls n'ont pas de pain ? Qu'ils mangent de la brioche ! „ était déjà attribuée, à l'identique ou avec des variantes, à des aristocrates dans l'Allemagne du 16e siècle, en Lettonie du $16^{\mathrm{e}}$ au $19^{\mathrm{e}}$ siècle et dans la France prérévolutionnaire à plusieurs dames de la cour telle Madame Victoire, fille de Louis XV, ou Madame du Barry, avant qu'elle ne se fixe sur l'épouse de Louis XVI ${ }^{12}$. De même, la fameuse phrase "Quand j'entends le mot “culture”, je sors mon revolver », écrite par un dramaturge nazi peu connu, Hans Johst, et reprise dans un discours par Baldur von Schirach, le chef des Jeunesses hitlériennes, a-t-elle ensuite été attribuée à des dignitaires plus en vue du Troisième Reich comme Joseph Goebbels et Hermann Göring.

Ainsi la convergence thématique d'anecdotes différentes permettra de brosser l'image d'un homme politique particulier, tandis que, de manière complémentaire, une même anecdote attribuée à plusieurs personnes révèlera l'image d'un type d'homme politique, à une époque donnée et dans une culture donnée.

\section{Le folklore narratif comme arme politique}

Il faut enfin toujours garder à l'esprit que la construction de l'image d'un homme politique par quelqu'un d'autre ${ }^{13}$ que lui-même et son équipe de conseillers en communication relève d'un jugement de valeur : l'anecdote a une fonction laudative ou, le plus souvent, péjorative. Les anecdotes, rappelle le Grand dictionnaire universel duxixe siècle, sont « des fictions qui dénaturent l'histoire pour faire ou défaire les réputations » (Larousse, 1866, p. 345, col.1). Ainsi le portrait de Mac-Mahon comme courageux militaire - proclamant fièrement «J'y suis, j'y reste » après avoir conquis une place forte durant la guerre de Crimée - a-t-elle été balayée par l'image négative de l'homme politique peu intelligent. La rumeur politique est un «contre-pouvoir» (Kapferer, 1990, p. 246). Les rumeurs sont utilisées entre adversaires politiques de camps adverses, mais aussi entre concurrents politiques du même bord, pour lesquels, l'attaque frontale n'étant pas possible, les rumeurs offrent l'avantage d'être une arme indirecte.

Les partisans de Bush ont contre-attaqué en exploitant la rumeur associant John Kerry à l’image des Français (Harsin, 2006) parce qu'il a le look d'un

12. Dans un contexte social de famine et d'émeutes populaires, l'anecdote est utilisée pour stigmatiser la bêtise ou l'arrogance des puissants (Campion-Vincent, Shojaei Kawan, 2002).

13. Comme la question de la véracité, la question de l'origine n'est pas primordiale pour l'étude des rumeurs. Qu'une rumeur ait pour source un individu malveillant ou, ce qui est le plus souvent le cas, une élaboration collective (voir Campion-Vincent et Renard, 2004), ce qui importe est la circulation d'une information ou d'un récit dans une population, sa signification et l'usage qui en est fait. 
Français, qu'il parle français, qu'il serait d'origine française (il est apparenté à Brice Lalonde) et qu'il est populaire en France. Mais c'est l'image négative du Français, principalement après que la France eut exprimé de fortes réticences contre la guerre en Irak, qui a été attribuée à Kerry : arrogant, bellâtre, élitiste, socialiste, lâche et gay. En inversant l'image, on obtient celle que ses partisans veulent donner de Bush, type du cow boy américain : modeste, naturel, démocratique, capitaliste, courageux et viril. Ce mécanisme de stéréotypage a été appelé «portraits contrastés» (Renard, 2007).

Parfois, une même anecdote brosse des portraits multiples. Ainsi, dans l'historiette ci-dessous, trois ministres d'Henri IV apparaissent comme les prototypes du courtisan aveugle, du conseiller prudent ou de l'homme de bon sens qui dit la vérité :

Henri IV, voulant faire connaître en un instant le caractère de ses différents ministres à un ambassadeur étranger, les fit venir successivement l'un après l'autre et leur dit: «Voilà une poutre qui menace ruine. » Villeroi, sans même lever les yeux, conseilla de la faire changer sur le champ. Jeannin, après avoir regardé avec attention, avoua qu'il n'en apercevait pas le vice, mais que pour ne rien risquer, il croyait prudent de la faire voir aux gens de métier. Sully vint ensuite, qui, consulté à son tour, répondit brusquement : "Sire, qui a pu vous donner cette terreur? Cette poutre durera plus que vous et moi. » (Grand dictionnaire universel duxixe siècle, 1866, p. 348, col. 1)

\section{Extensions possibles de l'approche}

Comme dans toute étude folklorique, on peut s'intéresser à l'enracinement des anecdotes contemporaines dans des motifs anciens. Ainsi la rumeur des "écoutes téléphoniques de l'Élysée» rappelle la légende de l'«oreille de Denys ", cet orifice dans la pierre qui permettait au tyran de Syracuse d'écouter sans être vu les conversations des prisonniers. Certains motifs touchent même au surnaturel : des légendes populaires prétendaient que Garibaldi, tel un moderne Moïse, a fait jaillir une source d'un rocher en y tirant un coup de canon, pour abreuver ses troupes assoiffées (Mannhardt, 1878, col. 568-569). Pensons aussi aux rumeurs de survie de personnalités politiques officiellement mortes (Renard, 2006b).

Il peut être également intéressant d'adopter une approche transversale, dans la mesure où des thèmes associés à des hommes politiques se retrouvent pour les artistes ou pour les savants : ainsi le génie précoce, la concurrence entre pairs, l'élève qui dépasse le maitre...

Enfin, une approche plus globale du champ politique conduirait à étudier aussi les anecdotes et histoires drôles ayant pour objet les partis ou les régimes politiques. 


\section{Références}

Allport Gordon W., Postman Leo J., 1965 [1945], «Les bases psychologiques des rumeurs », Psychologie sociale. Textes fondamentaux anglais et américains, A. Lévy éd., Paris, Dunod, p. 170-185.

BRUnVAND Jan H., 1993, The Baby Train and Other Lusty Urban Legends, New York, Norton.

CAmpion-VinCEnT Véronique, 1976, "Les histoires exemplaires », Contrepoint, n²2-23, p. 217-232.

CAMPION-VINCENT Véronique, RenARD Jean-Bruno, 2004, "Qui crée les rumeurs? De la thèse des individus malveillants à la théorie de la production collective», Les Cahiers du CREDAM, $\mathrm{n}^{\circ}$ 4, p.111-121.

CAmpion-Vincent Véronique, Shojaei Kawan Christine, 2002, "Marie-Antoinette et son célèbre dire. Deux scénographies et deux siècles de désordres, trois niveaux de communication et trois modes accusatoires ", Annales historiques de la Révolution française, $\mathrm{n}^{\circ}$ 327, p. 29-56.

CHRISTENSEn Arthur, 1925, Motif et thème. Plan d'un dictionnaire des motifs de contes populaires, de légendes et de fables, Helsinki, Suomalainen Tiedeakatemia.

FINE Gary Alan, 1985, "The Goliath effect : corporate dominance and mercantile legends ", Journal of American Folklore, $n^{\circ}$ 98, p. 63-84.

GAIDOz Henri, 1898-1899, "Légendes contemporaines», Mélusine, t. IX, col. 77-78 et 118. GRITTI Jules, 1978, Elle court, elle court, la rumeur, Ottawa, Stanké.

HARSIN J., 2006, «The rumor "John Kerry is French", i.e. haughty, foppish, elitist, socialist, cowardly and gay : anti-american », La France dans le regard des États-Unis, F. Monneyron et M. Xiberras éd., Perpignan, Montpellier, Presses universitaires de Perpignan, Publications de l’Université Paul-Valéry Montpellier 3, p. 323-354.

KAPFERER Jean-Noël, 1990 [1987], Rumeurs. Le plus vieux média du monde, Paris, Le Seuil. KRIS Ernst, KURz Otto, 1987 [1934], L'image de l'artiste. Légende, mythe et magie, trad. de l'anglais par M. Hechter, Paris, Rivages.

LAROUSSE Pierre éd., 1866, "Anecdote», Grand dictionnaire universel du XIXe siècle, Paris, Larousse et Boyer, t. 1, p. 344-350.

MANNHARDT Wilhelm, 1878, "Formation de mythes dans les temps modernes», Mélusine, t. I, col. 561-570.

NIETZSCHE Friedrich, 1951 [1879], La naissance de la philosophie à l'époque de la tragédie grecque, trad. de l'allemand par G. Bianquis, Paris, Gallimard.

RENARD Jean-Bruno, 2006a [1999], Rumeurs et légendes urbaines, Paris, PUF (Que sais-je ? $\left.\mathrm{n}^{\circ} 3445\right)$.

— 2006b, «Les rumeurs négatrices », Diogène, $n^{\circ} 213$, p. 54-73.

— 2007, "Le rôle des portraits contrastés dans la formation des stéréotypes », Stéréotypage, stéréotypes. Fonctionnements ordinaires et mises en scène, H. Boyer éd., t. II : «Identité(s) », Paris, L’Harmattan, p. 227-237.

RouQUETTE Michel-Louis, 1989, "La rumeur comme résolution d'un problème mal défini », Cahiers internationaux de sociologie, n 86, p. 117-122.

THOMPSON Stith, 1989 [1955-1958], Motif-Index of Folk-Literature, 6 vol., Bloomington, Indiana University Press. 